\title{
Percepção discente acerca das consequências do descarte inadequado dos resíduos de saúde
}

\author{
Discent perception about the consequences of improper disposal of health waste \\ Percepción discente sobre las consecuencias de la eliminación incorrecta de los resíduos sanitários
}

Recebido: 12/01/2021 | Revisado: 16/01/2021 |Aceito: 18/01/2021 | Publicado: 24/01/2021

\author{
Paulyne Souza Silva Guimarães \\ ORCID: https://orcid.org/0000-0001-8190-7330 \\ Universidade Federal de Alagoas, Brasil \\ E-mail: paulyne.guima@gmail.com \\ Rosana Quintella Brandão Vilela \\ ORCID: https://orcid.org/0000-0003-3910-4678 \\ Universidade Federal de Alagoas, Brasil \\ E-mail: zanavilela@gmail.com \\ Rillary Caroline de Melo Silva \\ ORCID: https://orcid.org/0000-0002-1488-1887 \\ Universidade Federal de Alagoas, Brasil \\ E-mail: melorillary@gmail.com \\ Paulo Jorge Torres Guimarães Silva \\ ORCID: https://orcid.org/0000-0001-9529-2752 \\ Universidade de Brasília, Brasil \\ E-mail: guima813@gmail.com \\ Esvaldo dos Santos Silva \\ ORCID: https://orcid.org/0000-0001-7388-3770 \\ Faculdade Estácio de Alagoas, Brasil \\ E-mail: es-valdosantos@hotmail.com \\ Alexandre de Souza Lima \\ ORCID: https://orcid.org/0000-0002-2634-4544 \\ Faculdade Estácio de Alagoas, Brasil \\ E-mail: enf.alexandresouzalima@gmail.com \\ Renné Costa da Silva \\ ORCID: https://orcid.org/0000-0001-6397-2163 \\ Universidade de Brasília, Brasil \\ E-mail: renneccosta@hotmail.com
}

\begin{abstract}
Resumo
Conhecer a percepção de discentes do curso de graduação em enfermagem acerca das consequências do manejo inadequado dos resíduos de saúde. Trata-se de uma pesquisa de campo descritiva e analítica com abordagem qualitativa, realizada com nove discentes do $4^{\circ}, 6^{\circ}$ e $7^{\circ}$ período. A coleta de dados foi realizada por meio da técnica de grupo focal. Os dados obtidos foram organizados e analisados resultando em três categorias, a saber: riscos socioambientais, riscos ocupacionais e gastos desnecessários. Constatou-se que o manejo inadequado dos resíduos de saúde está diretamente relacionado à produção de riscos na dimensão social, ambiental e ocupacional. Além de comprometer a dimensão econômica do setor saúde já tão precário. O tema requer uma reflexão ampliada e crítica, fazendo-se necessário que os discentes compreendam que tal ação negativa não se limita apenas a nível local, mas sobretudo global.

Palavras-chave: Resíduos de serviços de saúde; Gerenciamento de resíduos de serviços de saúde; Riscos ocupacionais; Saúde pública; Riscos ambientais.

Abstract

To understand the perception of undergraduate nursing students about the consequences of inadequate handling of sanitary waste. This is a descriptive and analytical field investigation with a qualitative approach, carried out with nine students from the 4th, 6th and 7th period. Data collection was performed using the focus group technique. The data obtained were organized and analyzed giving rise to three categories, namely: social and environmental risks, occupational risks and unnecessary expenses. It was found that the inadequate management of sanitary waste is directly related to the production of risks in the social, environmental and occupational dimensions. In addition to compromising the economic dimension of the already precarious health sector. The subject requires broad and critical reflection, so it is necessary for students to understand that such negative action is not limited only to the local level, but above all to the global level.
\end{abstract}


Keywords: Health Services waste; Health services waste management; Occupational risks; Public health; Environmental risks.

\begin{abstract}
Resumen
Conocer la percepción de los estudiantes de enfermería de pregrado sobre las consecuencias del manejo inadecuado de los desechos sanitarios. Se trata de una investigación de campo descriptiva y analítica con enfoque cualitativo, realizada con nueve estudiantes del $4^{\circ}, 6^{\circ}$ y $7^{\circ}$ período. La recolección de datos se realizó mediante la técnica de grupos focales. Los datos obtenidos fueron organizados y analizados dando lugar a tres categorías, a saber: Riesgos socioambientales, Riesgos laborales y Gastos innecesarios. Se encontró que el manejo inadecuado de los residuos sanitarios está directamente relacionado con la producción de riesgos en la dimensión social, ambiental y ocupacional. Además de comprometer la dimensión económica del sector salud ya tan precario. El tema requiere una reflexión amplia y crítica, por lo que es necesario que los estudiantes comprendan que dicha acción negativa no se limita solo a nivel local, sino sobre todo a nivel global.
\end{abstract}

Palabras clave: Residuos de servicios de salud; Gestión de residuos de servicios de salud; Riesgos laborales; Salud pública; Riesgos ambientales.

\title{
1. Introdução
}

O mundo enfrenta sérios desafios, entre os quais, a diversidade existente nos resíduos sólidos resultante das ações antrópicas do homem moderno (Moreschi, Rempel \& Backes, 2014). Partindo desse pressuposto, os resíduos de serviços de saúde, constituem um desafio com interfaces, dado a questão ambiental e os riscos para a saúde pública e do trabalhador (André, Veiga e Takayanagui, 2016).

Os resíduos de serviços de saúde representam um problema de saúde pública e ambiental em razão da presença de organismos patogênicos e/ou de suas toxinas (Moreira \& Gunther, 2016). O manejo segue um fluxo de ações para o gerenciamento adequado e seguro dos RSS. De acordo com a agência nacional de vigilância sanitária, é um processo articulado e contínuo constituído de dois momentos: o intraestabelecimento com as etapas de segregação, acondicionamento, identificação, armazenamento, coleta, transporte e tratamento interno; e o extraestabelecimento que compreende a coleta, transporte, tratamento externo e disposição final que depende da qualificação dos sujeitos envolvidos e embasamento técnicocientífico (Moreira \& Gunther, 2016).

Nessa realidade, os enfermeiros são responsabilizados pelo plano de gerenciamento de resíduos de serviços de saúde nas instituições de saúde, o que exige administração e gerenciamento de competências e habilidades (brasil, 2018). O plano de gerenciamento de resíduos de serviços de saúde é um documento norteador para a gestão, que envolve aspectos sanitários, ambientais, de saúde e segurança do trabalhador (Moreira \& Gunther, 2016).

Segundo Strohschoen, Moreschi e Rempel (2016), as formas tradicionais de ensino promovem uma diminuição da visibilidade dos discentes e egressos quanto aos aspectos ocupacionais e ambientais decorrentes do descarte inadequado dos resíduos por eles gerados.

Portanto, vale destacar as consequências do descarte inadequada dos resíduos de saúde: potencial de contaminação do solo, das águas superficiais e subterrâneas pelo lançamento dos resíduos em lixões ou aterros que por sua vez também proporcionam riscos aos catadores, incluindo aqui as crianças estão vulneráveis, principalmente por meio de lesões provocadas por materiais cortantes ou perfurantes, por ingestão de alimentos contaminados, ou aspiração de material particulado contaminado em suspensão (Silva, Sperling \& Barros, 2014).

Sendo assim, os resíduos resultantes da assistência à saúde humana (foco deste estudo), requerem manejo e gerenciamento adequados não só para atender a legislação vigente, mas, sobretudo para prevenir problemas relativos à saúde do trabalhador, saúde pública e meio ambiente.

Diante desse contexto, as diretrizes curriculares nacionais da enfermagem orientam o perfil do formando egresso com formação generalista capaz de atuar nos problemas/situações de saúde-doença, com senso de responsabilidade social e 
compromisso com a cidadania, como promotor integral da saúde do ser humano (Brasil, 2019). Além disso, o documento ainda descreve que o enfermeiro deve estar apto a fazer o gerenciamento e a administração tanto da força de trabalho quanto de recursos físicos, materiais e de informação.

No cenário geral, Brasil (2019) e Moreschi et al. (2014) advertem que o resíduos de serviços de saúde nem sempre são incorporados nos conteúdos teóricos e nas atividades práticas das instituições de ensino superior, podendo este profissional não reservar tempo para aprender a gerenciá-los, o que dificulta a consolidação de novos conceitos e consciência quanto ao descarte adequado. Sanchez et al. (2018) reforçam a necessidade de rever a trajetória do ensino na graduação em enfermagem, visando instrumentalizar os discentes para a questão dos resíduos, que representam, na atualidade, um dos mais intrincados problemas na dimensão social, econômica e ambiental.

$\mathrm{Na}$ intenção de responder a essa questão, o estudo teve como objetivo conhecer a percepção dos discentes de enfermagem acerca das consequências do descarte inadequado dos resíduos de saúde.

\section{Metodologia}

Trata-se de uma pesquisa de campo descritiva e analítica com abordagem qualitativa. Segundo Salbego et al. (2017), a pesquisa qualitativa busca compreender e interpretar a lógica interna dos participantes do estudo, conferindo-lhes o conhecimento de sua verdade, além de despertar a compreensão, a descrição e a análise da realidade por meio da dinâmica das relações sociais. Já para Pereira et. al (2018) para a utilização do método de pesquisa qualitativo é importante que haja interpretação por parte do pesquisador com suas opiniões sobre o fenômeno em estudo. Neles a coleta de dados muitas vezes ocorre por meio de entrevistas com questões abertas. O cenário de investigação refere-se ao curso de graduação em enfermagem de uma universidade pública do Nordeste.

Neste recorte do estudo, participaram nove discentes do $4^{\circ}, 6^{\circ}, 7^{\circ}$ período, nenhum dos participantes havia realizado qualquer atividade complementar que abordasse a temática dos resíduos de saúde. Os discentes foram recrutados de forma intencional, visto que os princípios de Biossegurança são abordados a partir do $2^{\circ}$ ano do curso e, especificamente, a temática em estudo no $3^{\circ}$ período.

Para alcançar o objetivo da pesquisa, utilizou-se a técnica de grupo focal com os discentes de enfermagem para a produção das informações. Segundo Taquette e Minayo (2015), o grupo focal constitui-se em uma estratégia de pesquisa adequada para a compreensão de experiências grupais e transformação da realidade, além de proporcionar uma relação de confiança entre o pesquisador e os participantes. Para nortear o grupo focal, foram utilizadas quatro questões disparadoras, os dados produzidos foram armazenados, transcritos, sistematizados, categorizado e analisado.

As informações coletadas foram analisadas utilizando-se a "análise de conteúdo, na modalidade temática, conforme as etapas preconizadas: pré-análise, exploração do material/codificação/descrição analítica e tratamento dos resultados obtidos/interpretação". Dessa análise, possibilitou a identificação de três categorias temáticas empíricas, apresentadas e discutidas nas sessões posteriores.

E é parte de um estudo mais abrangente desenvolvido pela autora para a obtenção do título de mestra, submetido ao Comitê de Ética em Pesquisa da Universidade Federal de Alagoas e aprovado pelo referido órgão, conforme Certificado de Apresentação de Apreciação Ética 02845918.50000.5013, Parecer no 3.099.358. O Termo de Consentimento Livre e Esclarecido e a autorização de concessão e uso da técnica de grupo focal foram assinados pelos participantes. Para preservar o anonimato dos discentes participantes, da instituição envolvida, definiu-se por apresentá-los da seguinte forma: representados pela letra D os discentes, seguida por um número, correspondente à ordem de sua participação no estudo (D1, D2, D3...D9). 


\section{Resultados}

Os discentes conseguiram descortinar três subcategorias: Riscos socioambientais, Riscos ocupacionais e Custos desnecessários.

Riscos socioambientais

O lixo de uma maneira geral pode afetar a água, o solo, o ambiente e a vida das pessoas (D2).

Enquanto profissionais da saúde temos que ficar mais atentos porque o lixo hospitalar pode agredir o planeta (D3).

Isso mesmo se desprezado sem tratamento o lixo pode prejudicar o ambiente e a população como um todo (D9).

Os discentes perceberam a relação existente entre os resíduos de saúde, a saúde humana e a saúde do meio ambiente, como também que a omissão dos profissionais da saúde quanto aos resíduos e isso acaba fazendo parte de um círculo vicioso, no qual o homem contamina o solo, a água, o meio ambiente em sua totalidade repercutindo em um desequilíbrio do planeta e, consequentemente o aparecimento de doenças.

Riscos ocupacionais

Os perfurocortantes quando manuseados de forma errada podem aumentar os riscos de acidentes de trabalho (D4).

Os resíduos podem contribuir para transmissão de doenças como o vírus do HIV e da Hepatite B para os trabalhadores da saúde (D5).

Hoje em dia mesmo com tudo que se diz, a respeito das medidas as serem tomadas um dos maiores acidentes dentro do hospital é com perfurocortante. Acho que deve ter preocupação com esse tipo de descarte, tirar o costume de tá reencapando seringa, respeitar o limite da caixa (D7).

Os discentes deram ênfase aos resíduos perfurocortantes, talvez em virtude das oportunidades teórico-práticas em manipular agulhas, cateteres, montar caixa de perfurocortante, entre outros. Descreveram os riscos que estes podem afetar a segurança e à saúde do trabalhador quando manuseados incorretamente. Para os discentes, não basta ter conhecimento é preciso ter boas práticas.

Gastos desnecessários

Posso dizer que o descarte impróprio dos resíduos contaminados para o hospital gera gastos (D2).

Acho que se não descartar da maneira certa aumenta o volume do contaminado e termina pagando mais (D7).

Os resíduos contaminados quando não tratados adequadamente podem contribuir para infecção hospitalar que para o paciente pode implicar em suspensão da alta do paciente (D8).

Os discentes vislumbram que o manejo inadequado dos resíduos de serviços de saúde implica em gastos, comprometendo desnecessariamente o orçamento da intuição e o agravamento da condição de saúde do paciente. Também percebem que caso a segregação não seja realizada na fonte, isso implica em aumento do peso dos resíduos infectantes requerendo tratamento prévio antes da disposição final.

\section{Discussão}

A questão ambiental tem se tornado um determinante do processo saúde-doença caracterizado por inúmeros problemas sanitários que afetam a população (KinalskI et al. 2017). 
Segundo Strohschoen, Moreschi e Rempel (2016) os cursos da área da saúde, principalmente o de curso de enfermagem, como ciência do cuidar, precisam unir forças entre ensino-serviço em prol da questão ambiental, visando despertar o mais precoce uma relação de mútua pertença entre o ser humano e a natureza.

Dessa maneira, é preciso refletir sobre os riscos sociais e ambientais versus as ações dos enfermeiros, ampliando os saberes, promovendo debates tanto na academia como no ambiente de trabalho. Buscando o tempo todo estratégias voltadas a reformulação das práticas em saúde.

Destaca-se que na década de 80, o Brasil promulgou a Constituição Federal que dedicou um capítulo ao meio ambiente. Assim, o Artigo 225, parágrafo $2^{\circ}$, inciso VI, dispõe sobre a obrigatoriedade do Poder Público em promover a Educação Ambiental em todos os níveis de educação, bem como promover a conscientização pública para a preservação do meio ambiente.

Petres e DA ROS (2018) reforçam que os impactos socioambientais extrapolam os muros das instituições de ensino na área de saúde, devendo os docentes conscientizar os futuros profissionais para disposição dos resíduos de saúde, uma vez que se descartados de forma inadequada atinge o meio ambiente, os recursos naturais e consequentemente a espécie humana.

Torna-se importante destacar, então, que a Educação Ambiental enquanto ciência tem por objetivo despertar no indivíduo a consciência crítica de que todos fazem parte do meio ambiente de forma que cada um venha desempenhar seu papel na preservação, conservação, manutenção dos recursos naturais e do meio ambiente, do qual é parte integrante.

Gomes et al.(2014) objetivaram discorrer sobre os impactos dos resíduos de serviços de saúde, desse modo, identificaram um distanciamento entre teoria e prática dentro e fora dos serviços, seja por parte dos gestores, profissionais de saúde e até mesmo pelos trabalhadores que manuseiam diariamente esses resíduos.

Para Bento et al., 2017, os profissionais de saúde estão expostos a riscos ocupacionais, entre os quais os riscos biológicos e químicos que podem causar infecções, doenças respiratórias, efeitos tóxicos, alérgicos, já Martins e Benito (2016) afirmam que os acidentes de trabalho na saúde mais graves relacionam-se a infecção pelo vírus da imunodeficiência humana, Hepatite B, Hepatite C representantes do maior impacto na saúde do trabalhador, caso não seja empregado nenhuma medida profilática.

Nesse ínterim, Cafure e Graciolli-Patriarcha (2015) afirmam que os acidentes de trabalho ocasionados por materiais perfurocortantes ou escarificante são frequentes, sobretudo quando não se utiliza caixas coletoras padronizadas e/ou quando não se obedece às instruções de preenchimento delas. A Agência Nacional de Vigilância Sanitária prediz que os resíduos resultantes dos perfurocortantes devem ser descartados em recipientes identificados, rígidos, providos com tampa, resistentes à punctura, ruptura e vazamento. Devem ser substituídos de acordo com a demanda do serviço ou quando o nível de preenchimento atingir 3/4 da capacidade, sendo proibidos seu esvaziamento manual e seu reaproveitamento (Brasil, 2018).

Compreende-se, então, que a segurança do trabalhador necessita ser contextualizada e problematizada na formação acadêmica de modo que os discentes e egressos sejam estimulados a analisar criticamente as ações que desenvolvem no cotidiano do trabalho, rompendo a dicotomia entre teoria e prática dos cursos da área da saúde, principalmente na graduação em enfermagem (foco deste estudo).

Quanto aos gastos desnecessários rios. Os autores (Zarpelão et al., 2018) enfatizam que adotando práticas responsáveis, certamente haveria uma redução de cerca de $80 \%$ nos gastos com tratamento e descarte dos resíduos, recursos que possibilitaram investir na melhoria do próprio sistema, na disponibilização de infraestrutura, nos processos de Educação Permanentes em Saúde entre outros.

O gerenciamento dos resíduos de serviços de serviços de saúde, não se configura apenas na dimensão econômica, mas uma questão ética de responsabilidade socioambiental, além de ser uma exigência legal. Se houver uma prática correta, haverá benefícios aos pacientes, à comunidade, ao meio ambiente e à segurança dos trabalhadores (Richardson et al., 2015). 
Um estudo que objetivou mapear e validar os subprocessos do manejo de resíduos no centro cirúrgico e calcular o custo dos materiais. Os autores Rosa, Mathias e Komata (2015) concluíram que a interligação entre os conhecimentos acadêmicos e metodologias baseadas no "pensamento enxuto" ainda precisa ser estruturada para refletir em práticas gerenciais sustentáveis no cotidiano profissional.

Dentro do contexto acima, verifica-se que as subcategorias repercutem os três pilares ou tripé da sustentabilidade - o social, ambiental e econômico. Esses elementos são prioridade para o desenvolvimento sustentável defendidas na Rio +20 que contribuíram para definição de uma agenda comum sobre o meio ambiente, com foco na economia verde e na equidade social.

\section{Considerações Finais}

Diante do exposto, observou-se que o objetivo desse trabalho foi alcançado, visto que permitiu conhecer a percepção dos discentes de graduação dos cursos de enfermagem. Contudo, cabe mencionar que essa abordagem do tema não teve a pretensão de explicar todos os riscos relacionados ao manejo inadequado dos resíduos de serviços de saúde, mas sim, contribuir para uma reflexão ampliada e crítica da prática em saúde, de modo que haja compreensão dos discentes que tal ação negativa não se limita apenas a nível local, mas de modo mais amplo, a nível global.

Nesse viés, novos estudos são necessários para maior aprofundamento da temática, envolvendo não só estudantes de enfermagem, mas também profissionais de saúde. Portanto, espera-se que este artigo possa contribuir com pesquisas futuras.

\section{Agradecimentos}

Ao Curso de Pós Graduação de Mestrado Profissional de Ensino na Saúde da Universidade Federal de Alagoas.

Aos discentes da Escola de Enfermagem da Universidade Federal de Alagoas, participantes da pesquisa.

\section{Referências}

André, S., Veiga T. \& Takayanagui A. (2016). Geração de Resíduos de Serviços de Saúde em hospitais do município de Ribeirão Preto (SP), Brasil. Engenharia Sanitária e Ambiental, 21(1), 123-130.

Bento, D. et al. (2017). O gerenciamento de resíduos de serviço de saúde sob a ótica dos profissionais de enfermagem. Texto Contexto - Enfermagem, 26 (1), e6680015.

Brasil. Ministério da Educação. Conselho Nacional de Educação. Câmara de Educação Superior. Resolução CNE/CES nº 3 , de 7 de novembro de 2001. Institui Diretrizes Curriculares Nacionais do Curso de Graduação em Enfermagem. Brasília, DF: MEC, 2001. http://portal.mec.gov.br/cne/arquivos/pdf/CES03.pdf

Brasil. Ministério da Saúde. Agência Nacional de Vigilância Sanitária. Resolução RDC n 222, de 28 de março de 2018 . Regulamenta as Boas Práticas de Gerenciamento dos Resíduos de Serviços de Saúde e dá outras providências. Brasília, DF: ANVISA, 2018. http://portal.anvisa.gov.br/documents/10181/3427425/RDC_222_2018_.pdf/c5d3081d-b331-4626-8448-c9aa426ec410.

Cafure, V. \& Graciolli-Patriarcha, S. (2015). Os resíduos de serviços de saúde e seus impactos ambientais; uma revisão bibliográfica. Interações, 16(2), 302314.

Cofen. Resolução 303, de 23 de junho de 2005. Dispõe sobre a autorização para o enfermeiro assumir a coordenação como responsável técnico do plano de gerenciamento de resíduos de serviços de saúde. Rio de Janeiro: COFEN, 2005. http://www.cofen.gov.br/resoluo-cofen-3032005_4338.html

Gomes C. et al (2014). Biossegurança e resíduos de serviços de saúde no cotidiano acadêmico. Revista de Ciências Farmacêutica Básica e Aplicada, 35 (3), 443-450.

KinalskI, D. et al (2017). Grupo focal na pesquisa qualitativa: relato de experiência. Revista Brasileira de Enfermagem, 70 (2), $443-448$.

Martins, D. \& Benito, L. (2016). Florence Nightingale e as suas contribuições para o controle das infecções hospitalares Universitas: Ciências da Saúde, 14(2), $153-166$.

Moreira A. \& Gunther W. (2016). Solid waste management in primary healthcare centers: application of a facilitation tool. Revista Latino-Americana de Enfermagem. 24 (e2768).

Moreschi C. et al (2014). A importância dos resíduos de serviços de saúde para docentes, discentes e egressos da área da saúde. Revista Gaúcha de Enfermagem. 35(2), 20-26. 
Research, Society and Development, v. 10, n. 1, e43610111915, 2021 (CC BY 4.0) | ISSN 2525-3409 | DOI: http://dx.doi.org/10.33448/rsd-v10i1.11915

Moreschi C., Rempel C. \& Backes D. (2014). A percepção de docentes de cursos de graduação da área da saúde acerca dos resíduos de serviços de saúde. Revista Baiana de Saúde Pública, 38 (3), 647-664.

Pereira, A. S., Shitsuka, D. M., Parreira, F. B., \& Shitsuka, R. (2018). Metodologia da pesquisa científica. Ed. UAB/NTE/UFSM. https://repositorio.ufsm. br/bitstream/handle/1/15824/Lic_Computacao_MetodologiaPesquisa-Cientifica.pdf.

Petres, A., \& Ros, M. (2018). A determinação social da saúde e a promoção da saúde. Arquivos Catarinenses de Medicina, 47 (3), $183-196$.

Richardson, J. et al. (2015). Nursing student's attitudes towards sustainability on health care. Nursing Standard, 29 (42), 36-41.

Rosa, C., Mathias, D. \& Komata, C. (2015). Custo De Gerenciamento De Resíduos De Serviços De Saúde (RSS): Estudo De Caso Da Unidade Deterapia Intensiva De Infectologia De Um Hospital Público Em São Paulo. Revista De Gestão Ambiental E Sustentabilidade, 4 (2), 127-143.

Salbego, C. et al (2017). Processo Educativo do enfermeiro frente ao gerenciamento dos resíduos de serviços de saúde. Biblioteca Lascasas, 13, 1-12.

Sanchez, A. et al (2018). Resíduos de Serviços de Saúde: conhecimento de enfermeiros da Atenção Básica. Revista Brasileira de Enfermagem, Brasília, 71(5), 2508-2517.

Silva, D., Sperling, E. \& Barros, R. (2014). Avaliação do gerenciamento dos resíduos de serviços de saúde em municípios da região metropolitana de Belo Horizonte (Brasil). Engenharia Sanitária e Ambiental, 19 (3), 251-262.

Strohschoen A., Moreschi C. \& Rempel C. (2016). A questão ambiental e a enfermagem: Percepções de Enfermeiros e Estudantes. Ensino, Saúde e Ambiente. 9(3), 297-309.

Taquette, S. \& Minayo, M. (2015). Características de estudos qualitativos conduzidos por médicos: revisão da literatura. Ciência \& Saúde Coletiva, 20 (8), $2423-2430$.

Zarpelão, R. et al (2018). Psychosocial Factors And Their Relation Between Work Accidents Among Outsourced Construction Workers. International Journal of Precious Engineering Research and Applications (IJPERA), 3 (1), 67-71. 